\title{
Le nuove sfide dell'epidemilogia in contesti politici e sociali
}

\section{Dalla sanità pubblica alle politiche di prevenzione}

\author{
Clelia Di Serio \\ Psychotherapie-Wissenschaft 8 (1) 65-73 2018 \\ www.psychotherapie-wissenschaft.info \\ CC BY-NC-ND \\ DOI: $10.30820 / 8242.12$
}

\begin{abstract}
Questo contributo è rivolto a fornire una panoramica ad ampio spettro su come l'evoluzione dell'informazioni biomediche abbia inevitabilmente portato ad un cambio di strumenti e modalità di analisi sia dal punto di vista dell'epidemiologia che della sanità pubblica. Per affrontare le sfide legate al cambiamento delle dinamiche di popolazione, ai nuovi grandi flussi migratori, al cambiamento delle dinamiche di invecchiamento, e all'evoluzione rapidissima della medicina nella direzione della «precision medicine» è necessario che la risposta socio-organizzativa del sistema sociale e sanitario si adegui tempestivamente ai mutamenti in corso e alle nuove esigenze. Nel l'articolo mostreremo come passando da una prospettiva individuale a quella di popolazione tipica dell'epidemiologia, cambino anche molti paradigmi legati ad una natura del dato, e ci si collochi in un modello tipo «global-system» in cui l'integrazione tra fonti diverse di informazione e interventi di prevenzione diventano imprescindibili.
\end{abstract}

Keywords: Public Health, surveillance, Ageing, big data, mental health

\section{Introduzione}

Esistono molti modi di introdurre i concetti fondamentali dell' epidemiologia e del suo ruolo nella società. Epidemiologia, sanità pubblica e politica sono sempre state legate fin da quando ci sono stati i primi tentativi di vaccinazione antivaiolosa attribuiti all'antica medicina indiana; $\mathrm{e}$ in particolare a Confucio che aveva emanato subito quelle che oggi chiameremmo «linee guida» sul modo di cuocere i cibi per tener lontane le malattie che 'entrano dalla bocca'. Ippocrate stesso descrisse con precisione il ruolo lassativo dell'orzo integrale rispetto a quello raffinato, privato della crusca, e la Scuola medica di Salerno individuò in alcune abitudini alimentari il fondamento per mantenersi in buona salute. Ma se vogliamo introdurre l'epidemiologia vera e propria bisogna riconoscere come questa non sia una disciplina "antica» come le altre scienze dure, ma legata all'evolversi della società.

Si puo' partire da un approccio di tipo storico integrativo che collochi la nascita della epidemiologia e della statistica medica in parallelo alla nascita e l'evoluzione di discipline con essa fortemente integrate come la genetica e l'informatica (Figura 1). Oppure considerare la nascita della epidemiologia come fortemente legata alle prime teorie importanti della probabilità nel seicento, da Pascal a Fermat (1654). Ma probabilmente il modo piu' corretto per collocare la nascita della disciplina dell'epidemiologia è di associarla alle prime indagini demografiche che portarono alla costituzione delle prime tavole della sopravvivenza da parte di uno dei fondatori della demografia John Graunt che nel 1662 scrisse «Natural and political observations mentioned in a following index» valutando la natalità, mortalità e distinzione fra sessi della popolazione londinese. Il lavoro fu poi portato avanti da De Moivre che nel 1725 introdusse e sviluppo' concetti come la censura e le prime funzioni di rischio lineari approfondite poi da Gompertz e Makeham che hanno implementato veri e propri modelli per il rischio istantaneo di morte. A lato dello sviluppo delle tecniche statistiche per l' analisi dei dati in termini predittivi nascono anche i primi modelli di diffusione delle malattie, come quello di John Snow, riconosciuto come il padre dell'epidemiologia, che nel 1854 grazie all'analisi spaziale dei dati di diffusione del colera a Londra riuscì a fermare l'epidemia identificando in uno dei due fornitori di acqua di Londra (Broad Street water pump) il responsabile dell'epidemia pur senza conoscere il Vibrio cholerae, e fermandola.

Qualunque sia l'approccio con cui ci si avvicina all'epidemiologia, piu' statistico-matematico oppure piu' medico-demografico, una cosa è certa: la disciplina comincia a svilupparsi solo a partire da metà del 1600 quando comincia a evidenziarsi un marcato aumento della «sopravvivenza» media dell'uomo. Solo a partire da tale momento storico comincia ad avere senso prima il concetto di "predizione», ovvero "vedere-prima» (prae-dicere, affermare in anticipo) quasi al confine con le discipline esoteriche, concetto che poi si trasforma in quello piu' matematico di «vedere prima» ovvero di «previsione» (prae videre, vedere prima) con i modelli previsivi lineari di Francis Galton e la teoria della regressione, fino ai giorni d'oggi, in cui tutto cio' che riguarda lo studio epidemiologico nelle discipline biomediche, dalla clinica alla ricerca di base, diventa fondamentale per la «prevenzione» (prar-venire, «arrivare prima») ovvero a 


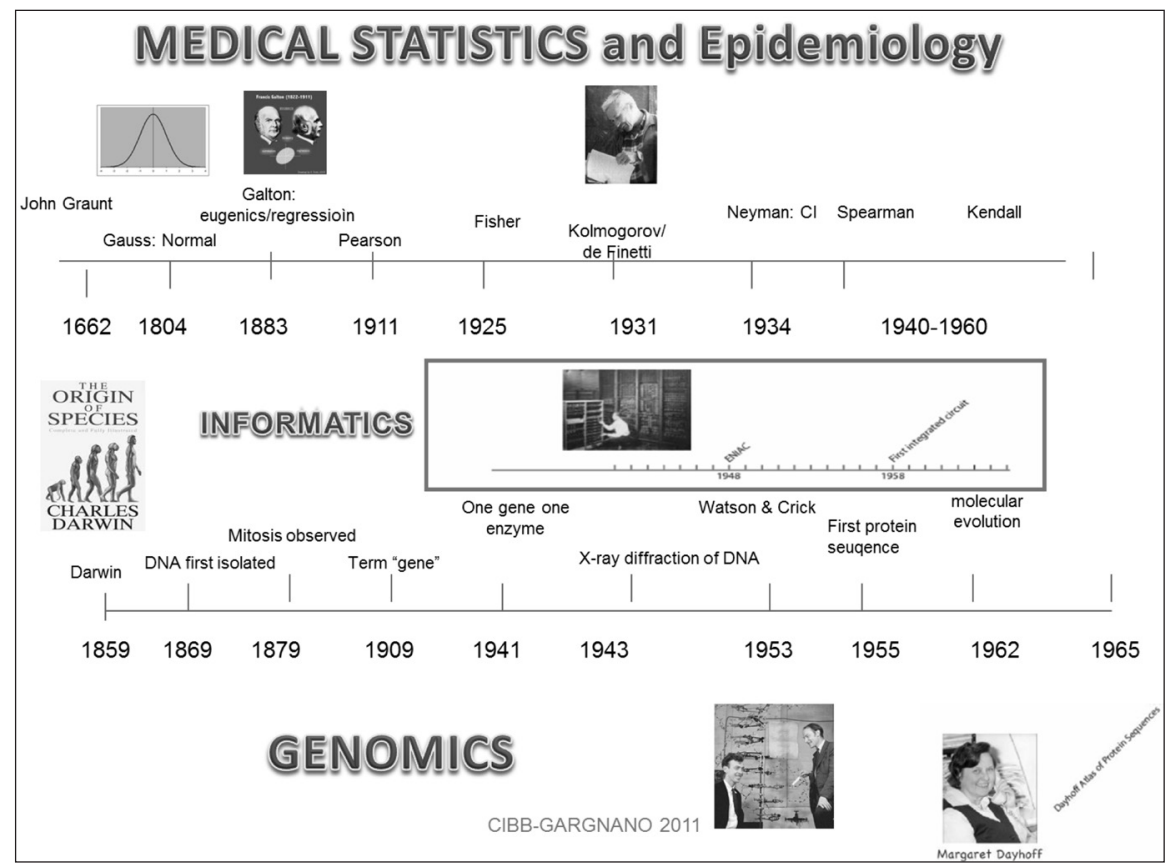

Figura 1: La figura mostra come la disciplina statistica si sia evoluta parallelamente e in modo integrato con discipline come la genomica e l informatica.

fini operativi e di intervento volti alla «early detection» delle malattie, che quindi rendono la disciplina immediatamente integrata con la società e con la salute pubblica,. Infatti il tentativo tentativo di evitare la comparsa di una malattia, o di una sua specifica manifestazione o di un suo aggravamento o recidiva fino a un possibile evento fatale, nonché il complesso delle procedure messe in atto a tale fine rappresentano aspetti fondamentali delle politiche di intervento sanitarie.

\section{Epidemiologia: una prospettiva individuale o di popolazione?}

Nella sanità pubblica si possono distinguere due prospettive che non sempre sono in accordo e il cui confronto puo' portare a conflitti etici e politici: una prospettiva individuale ed una prospettiva di popolazione. La prospettiva individuale focalizza sulla salute del soggetto, i fattori di rischio e di esposizione e sull 'esistenza di eventuali meccanismi di dipendenza tra questi fattori che possano essere causa di malattia nell'individuo. Malattie coronariche, tumore, infarto, ipertensione, asma, malattie neurodegenerative, tutti questi sono eventi potenzialmente catastrofici nella vita dell'individuo. La statistica medica in una concezione moderna si occupa di dare la base dell'evidenza alla medicina moderna: prima si è partititi con la «medicina traslazionale» (translational medicine), poi la cosiddetta «medicina personalizzata» (personalized medicine) e oggi invece si parla di «medicina di precision» (precision medicine). L'evoluzione della «tecnologia del dato» ha avuto come conseguenza anche la generazione di nuovi processi di interazione tra epidemiologia, sorveglianza, prevenzione e sanità pubblica come illustrato dalla figura 2 .
Cosa c'è sotto questo continuo cambio di prospettiva della medicina e co me la statistica medica ha cambiato i suoi paradigmi conseguentemente?

Il punto fondamentale è il cambio del tipo di dato che ha portato il mondo della biomedicina a diventare un unico «sistema globale» (global system). Fino a pochi decenni fa il problema dell'utilizzo della statistica in medicina era un problema di «fornire evidenza» attraverso un processo puramente di «induzione» ovvero un processo inferenziale in cui, partendo da un campione anche relativamente piccolo, tramite gli strumenti dell'inferenza statistica si arrivasse a «fare inferenza» sulla popolazione per poter parlare di «evidence based medicine» (EBM). Oggi, nell'epoca dei BIG DATA, di cui parleremo nell'ultima sezione, il paradigma della statistica in medicina si è invertito e la medicina si è trasformata in un sistema integrato di informazioni. Con l'avvento della genomica, della proteomica, dei grandi consorzi e del federalismo dei dati biomedici, si apre una prospettiva piu' strettamente «deduttiva» della disciplina. Infatti la «medicina traslazionale» si occupa di mettere insieme fonti di dati eterogenee e conoscenze interdisciplinari, fruibili nella ricerca di base e nella clinica, e di integrarle in modo trasversale per migliorare la conoscenza delle patologie per inizializzare un processo di «deduzione» in cui dalla cura generale si cerca di tornare indietro ad una terapia "personalizzata» e calibrata sul paziente. Tuttavia nell'accezione moderna di medicina si preferisce parlare di «precision medicine» per ritrovare una dimen-

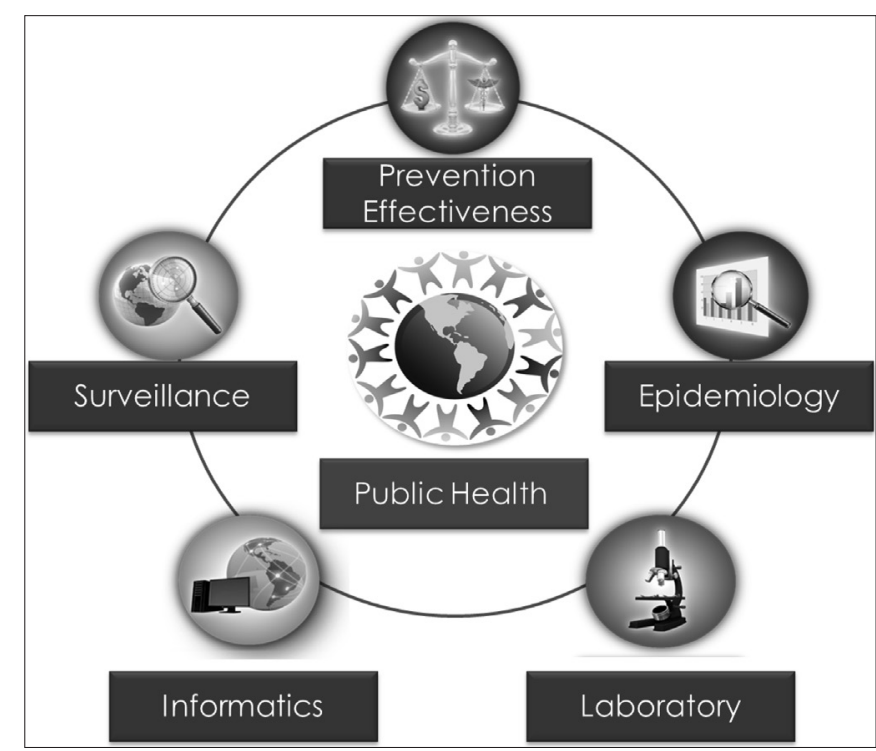

Figura 2: Processo di interazione tra discipline in sanità pubblica. 
sione piu' allargata rispetto alla medicina personalizzata, dopo che anche le agenzie piu' autorevoli, come National Research Council, hanno verificato che da un punto di vista della sostenibiltà del sistema sanitario pensare ad una terapia brevettata unicamente per le caratteristiche di un individuo risulta anti-economico e anche a tratti poco etico. Oggi si preferisce parlare di «precision medicine» ovvero di "precisione» in cui le terapie vengono brevettate sulla base di fattori genetici, ambientali e di «lifestyle» per giungere a delle terapie che siano adattabili ad un «gruppo» di pazienti che le condividono piuttosto che all'individuo singolo. E' possibile tuttavia comprendere come la prospettiva «individuale» potrebbe portare a interventi a livello di sanità pubblica che sono molto diversi rispetto a quelli identificati da una "prospettiva di popolazione» come illustrato in Figura 3.

Il senso autentico della epidemiologia, e che la distingue dalla statistica medica, risiede nella sua etimologia ovvero «epi-demo-logos», studio della popolazione . La prospettiva di popolazione si focalizza sulla una patologia rispetto ad un grande numero di persone, fino ad estendersi alle cosiddette epidemie di massa. L'esposizione a fattori di rischio viene valutato all'interno delle esposizione di gruppi di individui.

Nella Figura 4 vengono rappresentati i tassi di decesso per sette tipi di tumore [colon \& rectum, leukemia, liver, lung \& bronchus, pancreas, prostate, and stomach] nei maschi degli Stati Uniti nel un periodo dal 1930 al 2005. Cosa leggiamo in un grafico di questo tipo tipico dell'epidemiologia? Da una parte vediamo l'andamento di sette tipi di tumore nel tempo come in tutti i grafici longitudinali dove a fronte della stabilità di quasi tutti i tumori si vede il drammatico incremento del tumore al polmone e in particolare si puo' individuare un change point.

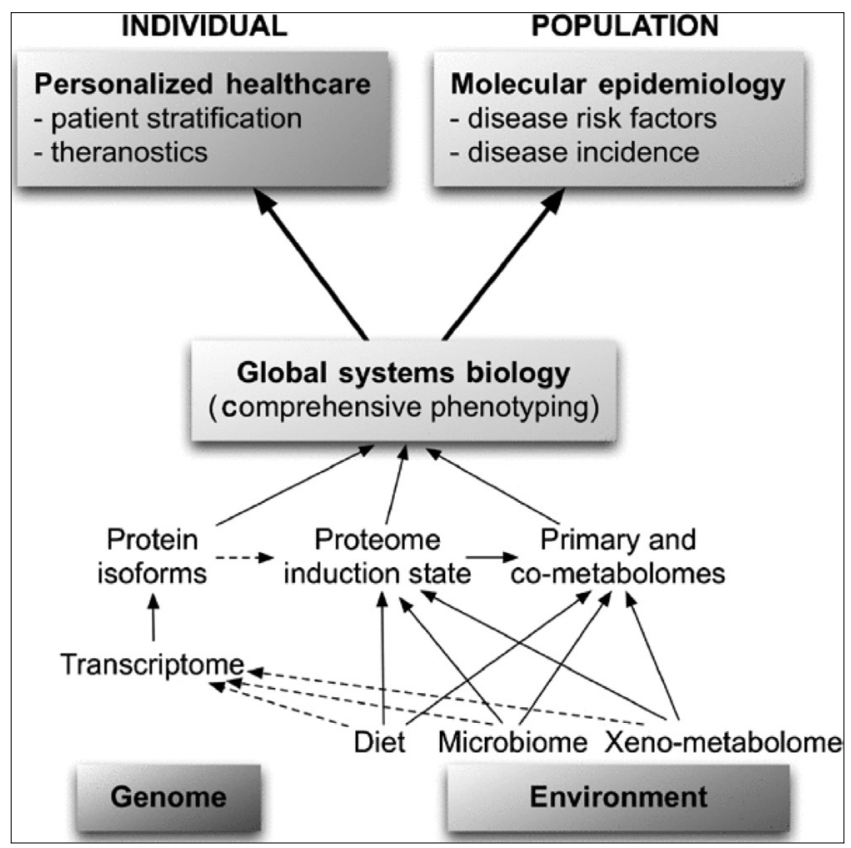

Figura 3: Relazioni tra systems biology, personalized healthcare e molecular epidemiology.

Vediamo che il picco del 1965 in una prospettiva epidemiologica risulta di lettura fondamentale per la ricerca delle cause dell'incremento. In quegli anni l'escalation del tabacco è stato rilevato dalla WHO come «the most tragic developments in the modern history of medicine» (Jan Stjernsward (WHO)). Da allora fino ai giorni nostri è iniziata una campagna contro il fumo a livello di società mondiale, partendo dal divieto di pubblicità esplicita, all'inserimento di frasi dissuasorie sulle sigarette stesse per arrivare a rendere obbligatorio il divieto di fumo in ogni luogo pubblico. Il tumore al polmone rappresenta una

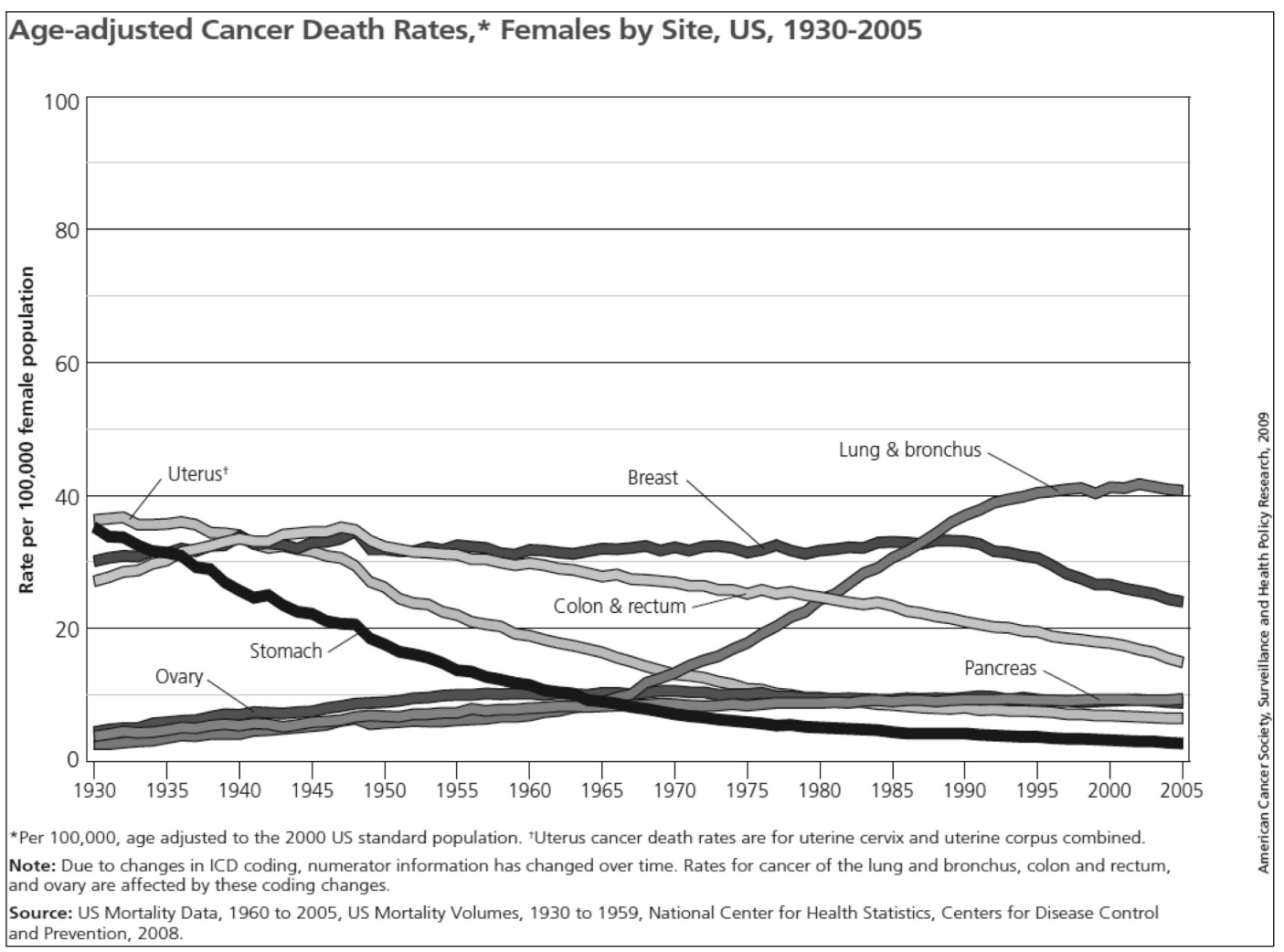

Figura 4: American Cancer Society's publication Cancer Facts \& Figures and is based on data from the National Center for Health Statistics and the Bureau of the Census. 
chiara dimostrazione di «epidemia di massa» del bisogno di una prospettiva di popolazione per arginarla. Quindi la prospettiva di popolazione non indaga tanto i fattori di rischio clinico-biologico per il soggetto, riferendosi piuttosto a fattori ambientali e a conseguenti modifiche nel sistema politico sanitario.

Un altro esempio di fenomeno pandemico del 20th viene rappresentato dall'HIV/AIDS (immunodeficiency virus e acquired immunodeficiency syndrome). In una prospettiva individuale su HIV l'istituzione si focalizza nel prevenire e nell'evitare comportamenti rischiosi negli individui, per cui il "Centers for Disease Control and Prevention (CDC)» ha investito molti milioni di dollari per programmi educativi inclusivi dell'acquisto di test HIV da distribuire a livello largo di popolazione. In una prospettiva di popolazione in contrasto, ci si basa su elementi socio-economico e demografici che hanno contribuito a diffondere il virus quali (1) il flusso migratorio di lavoratori da aree ad alto tasso di pandemia- come il sudest sub-sahriano (2) sudditanza delle donne africane all'interno delle tribù per cui fin da età infantile sono costrette a comportamenti promiscui che aumentano abbondantemente il rischio di malattia (3) l'esplosione

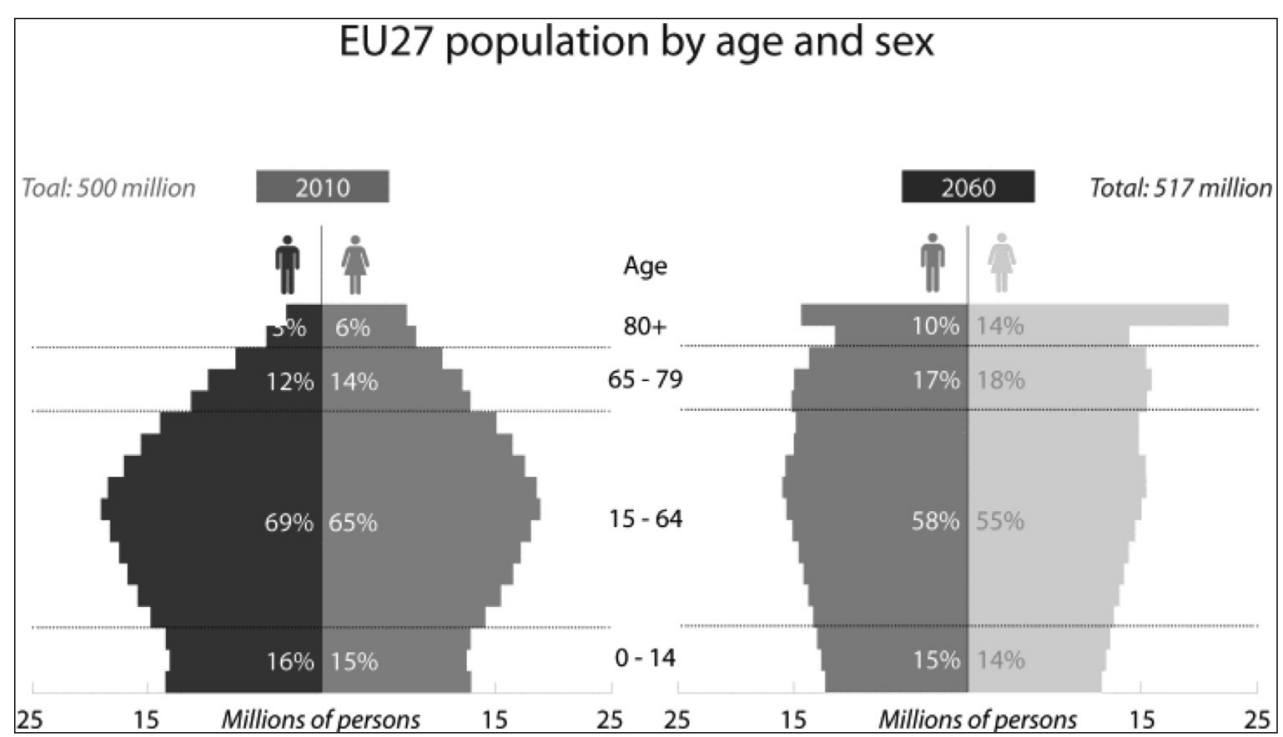

Figura 5: Population growth stratified by gender. piu' elevato nelle popolazion con rapida crescita della vita media.

Il rapporto di Eurostat 2010-2060 mostra una dinamica di crescita molto rapida come si puo' osservare nella figura 5 con un particolare picco evidente per le donne anziane previste in grande aumento. Sebbene il peso dello sviluppo socio-economico gravi in gran parte sulla donna, le politiche di intervento sono ancora poco differenziate per gender, e tardano ad adeguarsi a fronte del maggiore impegno delle donne nel mercato del lavoro, al di fuori famiglia, e per l'indebolimento del tessuto familiare e parentale che porta le donne a dover accudire prima i figli e poi spesso contemporaneamente il marito e i genitori. del traffico aereo e la globalizzazione piu' in general che hanno alla rapida disseminazione del virus. La prospettiva individuale si basa sulla «diagnostica» e porta quindi alla ricerca del trattamento «tailored» sul paziente. La prospettiva di popolazione si occupa di "screening», prevenzione di massa, ricerca del livello di intervento primario e secondario della prevenzione.

\section{Epidemiologia dell'Ageing e Surveillance}

Il processo di Ageing appare in certe fasi di sviluppo della popolazione, ed è il risultato di un calo della fertilità simultaneo ad un aumento della sopravvivenza e delle aspettative di vita. Questi cambiamenti nella composizione della popolazione per quanto prevedibili sono avvenuti con maggiore velocità rispetto alle attese tanto da non sempre consentire al sistema socio-economico di adeguarsi con efficienza alla trasformazione e pertanto sono stati recepiti dalla maggioranza delle istituzioni come fenomeni negativi, tanto da essere considerati come il «problema dell'invecchiamento» per lungo tempo. A partire dagli anni '70 questa visione è lentamente mutata, soprattutto grazie al demografo Edward Rosset che nel 1978 condusse una ricerca che dimostrava uno sviluppo 


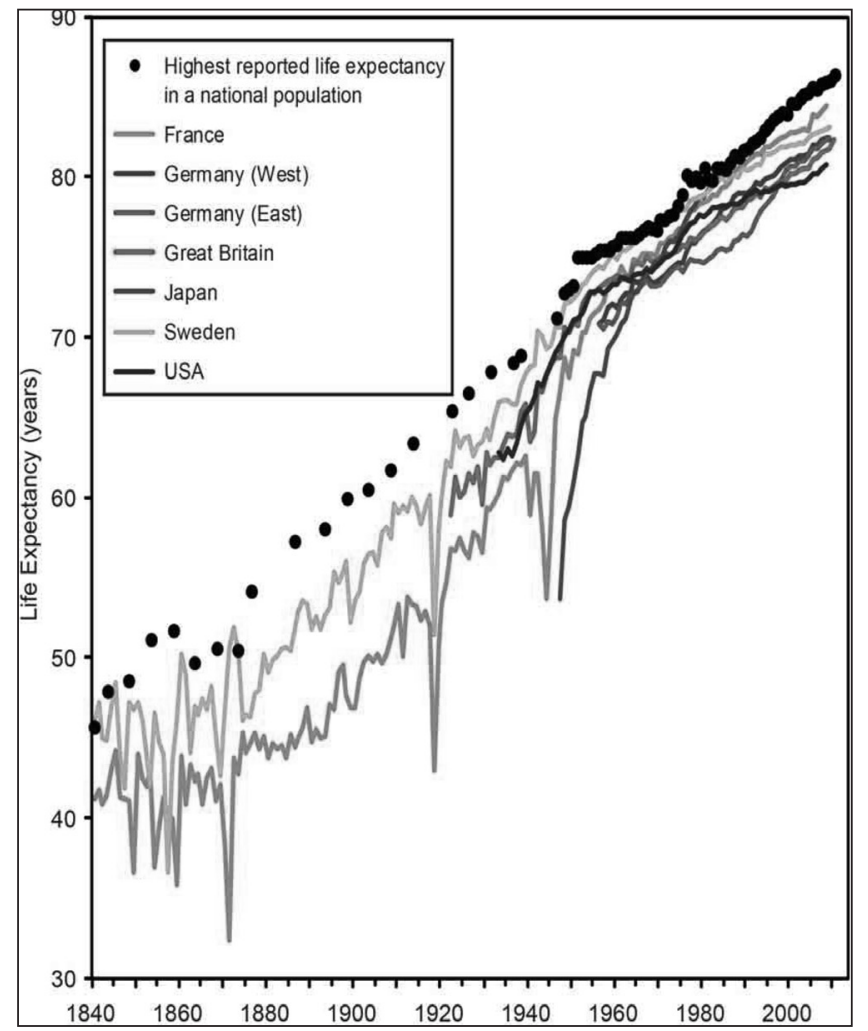

Figura 6: Source: Highest reported life expectancy for the years 1840 to 2000 from online supplementary material to Oeppen J, Vaupel JW. Broken limits to life expectancy. Science 2002: 296.

Eurostat ha rilevato come la maggioranza degli stati europei non siano ancora predisposti a politiche di «active ageing» ovvero politiche in cui l'anziano qualora in buone condizioni di salute possa diventare parte attiva della società, e non solo passiva, partecipando laddove possibile a tutte le attiviità economiche, culturali e sociali della società che devono essere quindi diversamente targettate.

Il carattere globale e le enormi implicazioni del fenomeno richiedono risposte coordinate da parte dei vari Stati, al fine di promuovere politiche intersettoriali che riguardano un ampio spettro di questioni sociali ed economiche.

\section{Il ruolo della surveillance nella società dei disturbi mentali}

Quando parla di «surveillance» non si puo' non considerare il ruolo cruciale della sanita pubblica nel promuovere la salute "psicologica» alla base di considerazioni inerenti la qualità della vita, la sostenibilità di un sistema socio-economico, e la salute pubblica in generale. I problemi di salute mentale sono diventati infatti una delle principali cause di assenteismo sui luoghi di lavoro e di pensionamento anticipato in Europa. Inoltre, l'attuale recessione economica e i suoi effetti sul mercato del lavoro aggiungono problemi all'occupazione e alla qualità della vita delle persone che soffrono di disturbi mentali e delle loro famiglie. La pubblicazione dell'Oms Europa «Mental health and well-being at the workplace - protection and inclusion in challenging times» Infatti le malattie mentali nel mondo affliggono circa 450 milioni di persone, considerando che un quarto della popolazione mondiale si stima che svilupperà un disturbo mentale o comportamentale in qualche momento della propria vita. Le patologie mentali pesano circa per il $25 \%$ delle disabilità negli stati uniti in Canada e Europa occidentale e, se includiamo anche le patologie cognitive, rappresentano una tra le cause principali di morte prematura.

Per esempio negli Stati Uniti si stima una prevalenza durante la vita della popolazione adulta di circa il $29 \%$ per disturbi di ansia e depressione, il $25 \%$ per disturbi da perdita di controllo e OCD, $21 \%$ disturbi dell umore e altri disturbi al $46 \%$. Gli effetti dei disturbi mentali sono evidenti nel corso della vita a qualunque livello socioeconomico. Anche il costo per il sistema sanitaro è enorme. In US si valuta un costo stimato annuale di \$150 billione escludento I costi della ricerca.

La figura successiva vuole visualizzare il rapporto tra prevalenza e trattamento in uno dei maggiori disturbi psichici, ovvero la depressione.

In Europa i problemi di salute mentale rappresentano quasi il $20 \%$ del totale delle malattie e colpiscono addirittura una persona su quattro nell'arco della propria vita. Dei dieci Paesi con i più alti tassi di suicidio nel mondo, nove si trovano in Europa. Nonostante questi dati, molto spesso i servizi sanitari che vengono offerti non rispon-

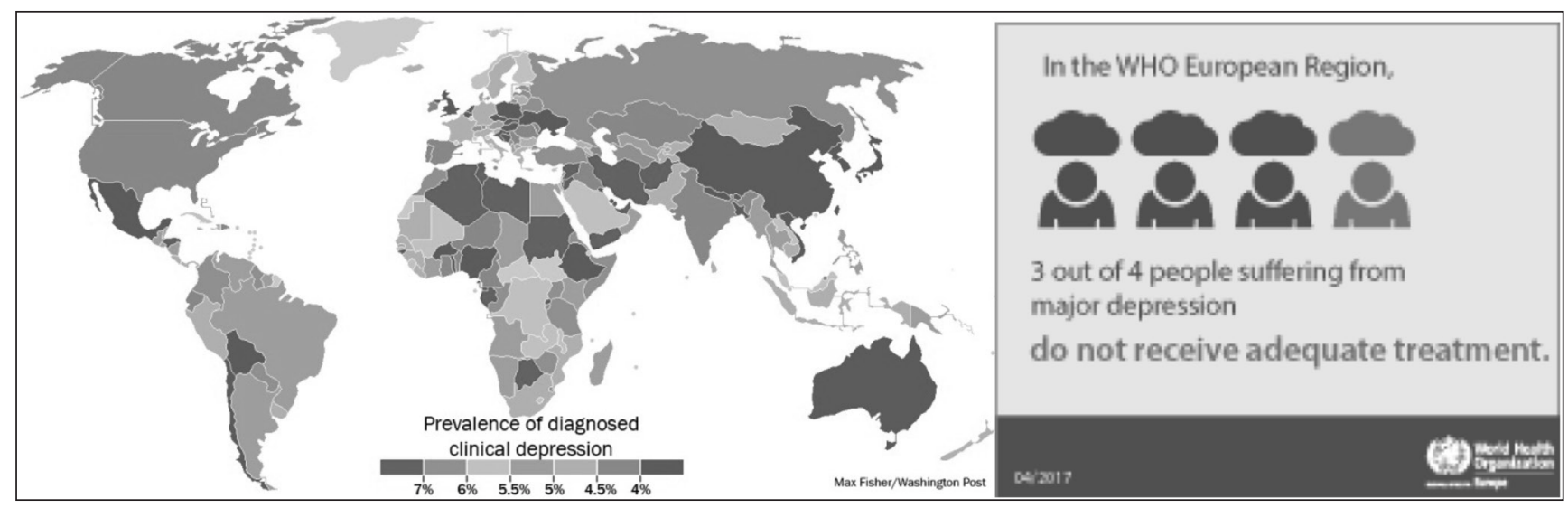

Figura 7: Prevalence in diagnosis of mental disorders versus lack of treatments. 
dono alle necessità e alle esigenze dei pazienti: il gap tra il tipo di cure di cui i malati avrebbero bisogno e quello che invece ricevono è ancora molto ampio. Per esempio, la metà di coloro che soffrono di depressione non viene mai curata e, più in generale, anche in Paesi dotati di un sistema sanitario sviluppato il $44-70 \%$ di coloro che soffrono di malattie mentali non è sottoposto ad alcuna terapia (figura 7). In un quarto degli Stati europei non sono neanche disponibili i servizi di assistenza e in alcuni Paesi oltre il $50 \%$ dei pazienti viene ospitato in strutture troppo grandi e inadeguate.

Come riporta il rapporto dell WHO 2013-2020 sulla prevenzione in temi di salute mentale ( $(\mathrm{http} / / / \mathrm{www} . e u r o$. who.int/en/health-topics/noncommunicable-diseases/mental-health/policy) le persone con disturbi mentali sperimentano tassi di disabilità e di mortalità notevolmente più elevati rispetto alla media. Per esempio persone con depressione maggiore e schizofrenia hanno una possibilità del 40-60\% maggiore rispetto al resto della popolazione di morte prematura, a causa di problemi di salute fisica, che spesso non vengono affrontati (come cancro, malattie cardiovascolari, diabete o infezione da HIV), e di suicidio. Esso è la seconda causa di mortalità nei giovani su scala mondiale. 12 Spesso i disturbi mentali influiscono su altre malattie, quali ad esempio il cancro, le malattie cardiovascolari e l'infezione da HIV/AIDS, e sono a loro volta influenzate da queste; e pertanto necessitano di servizi comuni e un'attiva mobilitazione di risorse. Per esempio, è stato infatti dimostrato che la depressione pu causare una predisposizione all'infarto del miocardio e al diabete; i quali a loro volta aumentano il rischio d'insorgenza di una depressione. Si è constatato inoltre che spesso i disturbi mentali si accompagnano ai disturbi da uso di sostanze psicoattive. Nel loro complesso, i disturbi mentali, neurologici e da uso di sostanze psicoattive, comportano costi elevati, che ha raggiunto il $13 \%$ del carico globale di malattia su scala mondiale nel 2004. La depressione da sola rappresenta il $4,3 \%$ del carico globale di malattia ed è una delle principali cause di disabilità a livello mondiale ( $11 \%$ degli anni di vita vissuti con una disabilità nel mondo intero), particolarmente nelle donne. Le conseguenze economiche di queste condizioni di compromissione della salute sono altrettanto importanti: un recente studio ha stimato che l'impatto cumulativo dei disturbi mentali a livello mondiale in termini di perdita della produzione economica ammonterà a 16.300 miliardi di dollari tra il 2011 e il 2030.

Il primo studio epidemiologico sulla prevalenza dei disturbi mentali rientra nel progetto europeo European Study on the Epidemiology of Mental Disorders (ESEMeD), al quale hanno preso parte sei paesi (Italia, Belgio, Francia, Germania, Olanda e Spagna). Lo studio fornisce un quadro della frequenza dei disturbi mentali nella popolazione italiana, delle relazioni tra di loro, dell'associazione con i possibili fattori di rischio e dei trattamenti ricevuti.(https://www.ncbi.nlm.nih.gov/pub$\mathrm{med} / 15128382$ )

Questo e altri progetti sono stati realizzati nell'ambito della WHO World Mental Health (WMH) Survey
Iniziative, al quale partecipano più di 30 paesi diversi; questo ampio progetto di ricerca epidemiologica è stato promosso congiuntamente dall'O.M.S. e dall'Università di Harvard (http://www.hcp.med.harvard.edu/wmh/). A livello europeo il progetto è stato finanziato dall'Unione Europea e dalla GlaxoSmithKline.

In Svizzera il 27\% della popolazione adulta (18-65) ha sperimentato almeno uno di una serie di disturbi mentali nell'ultimo anno(includendo depressione, ansia, disordini dell alimentazone, dipendenza da sostanze). I tassi sulle donne sono leggermente piu' alti di quelii sugli uomini per quasi tutti i disturbi tranne la dipendenza da sostanze ( $5.6 \%$ uomini; 1.3 nelle donne). I valori di prevalenza in Svizzera di queste patologie anche più alti rispetto alla media europea si combina tuttavia ad un sistema sanitario federale molto efficiente ed orientato all'accessibilità dei servizi sanitari, che rende la Svizzera al primo posto anche integrandola con l'Europa per l'accessibilità dei servizi e i risultati sulla salute secondo l' 'Euro Health Consumer Index , che ogni anno classifica i sistemi sanitari europei. Per questo motivo la Svizzera vanta uno dei piu' alti numeri di psichiatri in Europa, pari a 30 per 100000 abitanti, laddove la mediana europea è pari a 9.

Risulta quindi prioritario considerare la salute mentale come un problema urgente ed integrale in tutto il mondo che dovrebbe essere trattato dalle istituzioni con la stessa urgenza di qualunque altro disturbo fisico, dato che le determinanti sono state bene identificate come di puo' vedere dalla Figura 8.

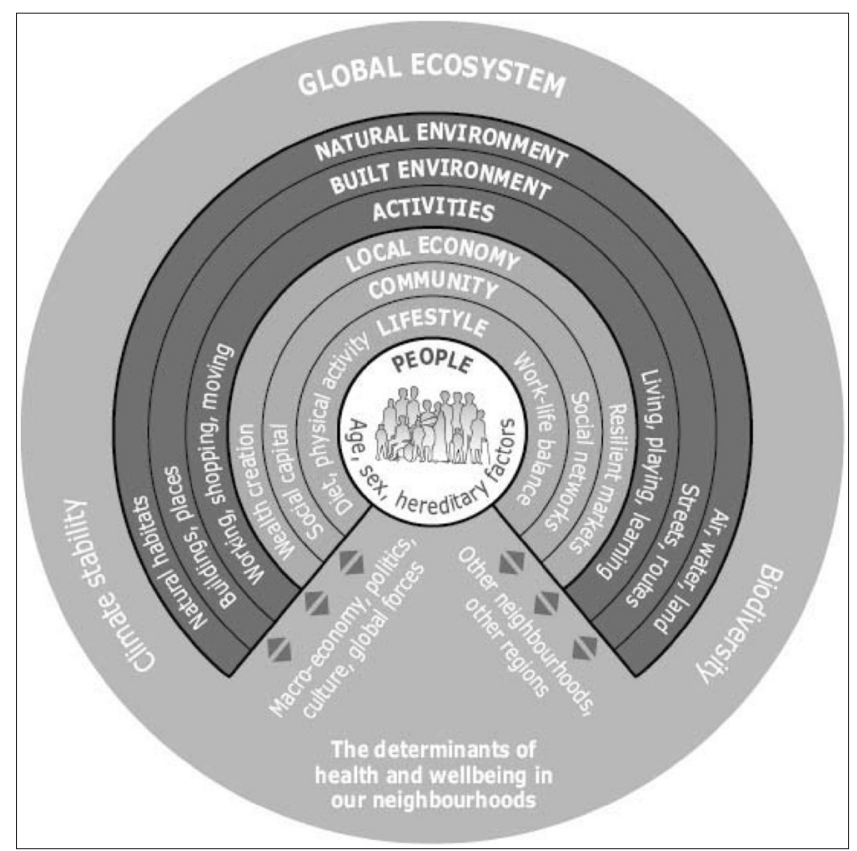

Figura 8: (fonte Barton and Grant). Determinants of health and wellbeing.

I disturbi mentali inoltre presentano co-morbidità con altre patologie in quanto inducono comportamenti dannosi per la salute, come l'abuso di sostanze, l'abuso di droga e alcool e la mancanza di attività fisica. La depressione oggi si conosce come uno dei fattori di rischio maggiori per l'ipertensione, le malattie cardiovascolari, e anche 
per il diabete causato da disturbi alimentari. Per poi non parlare di tutti i problemi connessi alla psicologia della salute, ovvero evidenze di mancata risposta ai trattamenti (oncologici, cardiaci) dovuta principalmente alla depressione post operatoria. Lo scarso livello di literacy che continua ad esistere circa le malattie mentali e il pregiudizio culturale prevalente fa si' che nonostante esistano trattamenti efficaci per molte di queste malattie la maggior parte delle persone con diagnosi non ricorre ai trattamenti. Questi aspetti socio-culturali molto specifici dei disordini mentali producono effetti devastanti delle mancate diagnosi per cui patologie che sarebbero leggere qualora rilevate e curate con efficacia si cronicizzano. Una delle sfide principali per la sanità pubblica diventa quindi l'identificazone dei fattori di rischio, l'aumento di consapevolezza e di literacy sull'efficacia dei trattamenti, la rimozione dello stigma associato ancora oggi in molti paesi ai trattamenti psicologici, l'aumento della disponibilità del personale medico sostenibile dal sistema sanitario per la prevenzione primaria e secondaria o la diagnosi precoce soprattutto in quelle popolazioni che ne sono affette in modo sproporzionato.

Le istituzioni di sanità pubblica è necessario incorporino dei programmi di prevenzione per i disturbi mentali nella surveillance generale delle malattie croniche per essere in grado di affrontare $\mathrm{i}$ alcuni aspetti principali rilevati nei report del OMS (WHO)

1. Identificazione dei soggetti affetti da disturbi mentali (95\% dei paesi)

2. Diagnosi precoce dei problemi $(86 \%)$;

3. Capacità di trattare regolarmente i soggetti affetti delle malattie che coinvolgono il sistema nervoso e lo stato di disabilità psichica $(85 \%)$

4. fornire servizi di salute mentale e di supporto sociale comprensivi, integrati e capaci di risposta a livello territoriale

5. rafforzare i sistemi informativi, le evidenze scientifiche e la ricerca per la salute mentale

Alla luce di queste considerazioni il piano di azione globale 2013-2010 mette in luce come sia importante spostarsi verso un sistema sanitario n cui la salute mentale venga valorizzata, promossa e protetta, nel quale i disturbi mentali siano prevenuti e le persone affette da questi disturbi siano in grado di esercitare appieno tutti i diritti umani e di accedere in tempo utile a servizi di cura sanitari e sociali di alta qualità e culturalmente appropriati che promuovano la recovery, affinché possano ottenere il più alto livello possibile di salute e di partecipare pienamente alla vita sociale e lavorativa, libere da stigma e discriminazione.

\section{Precisione, prevenzione: dai big data alla salute individuale}

Nella società moderna l'evoluzione della tecnologia di produzione del dato biomedico ha spostato l'attenzione sulla «grande informazione» derivabile dai «BIG DATA».
La biostatistica oggi rientra nella piu' ampia classe disciplinare di «data science». I progressi tecnologici e biomedici hanno creato un flusso dei dati biologici e di salute pubblica in rapida e continua crescita, generando una robusta base di conoscenze potenzialmente in grado di migliorare l'assistenza sanitaria grazie ad approcci più efficaci e personalizzati per la prevenzione, la diagnosi e il trattamento delle malattie e alla possibilità di rendere più efficiente l'erogazione dei servizi di salute e sostenere l'innovazione e la crescita economica. I BIG DATA si riferiscono al volume dell'informazione, alla sua complessità e alla velocità di fruizione della stessa. Infatti i «grandi» dati biomedici vengono classificati in base alle cosiddette 3V : «Volume», che si riferisce al numero di campioni e al numero di variabili e caratteristiche per campione. Questo aspetto riguarda per esempio la creazione di grandi consorzi internazionali di raccolta di dati biomedici che coinvolgono diverse istituzioni di sanita', ospedali e centri di ricerca; "Velocity» che si riferisce alla velocità di generazione dei dati legata alle nuove tecnologie e «Variety» che riguarda le fonti differenti di dati come dati clinici, genomici e biomolecolari come illustrato nella Figura 9.

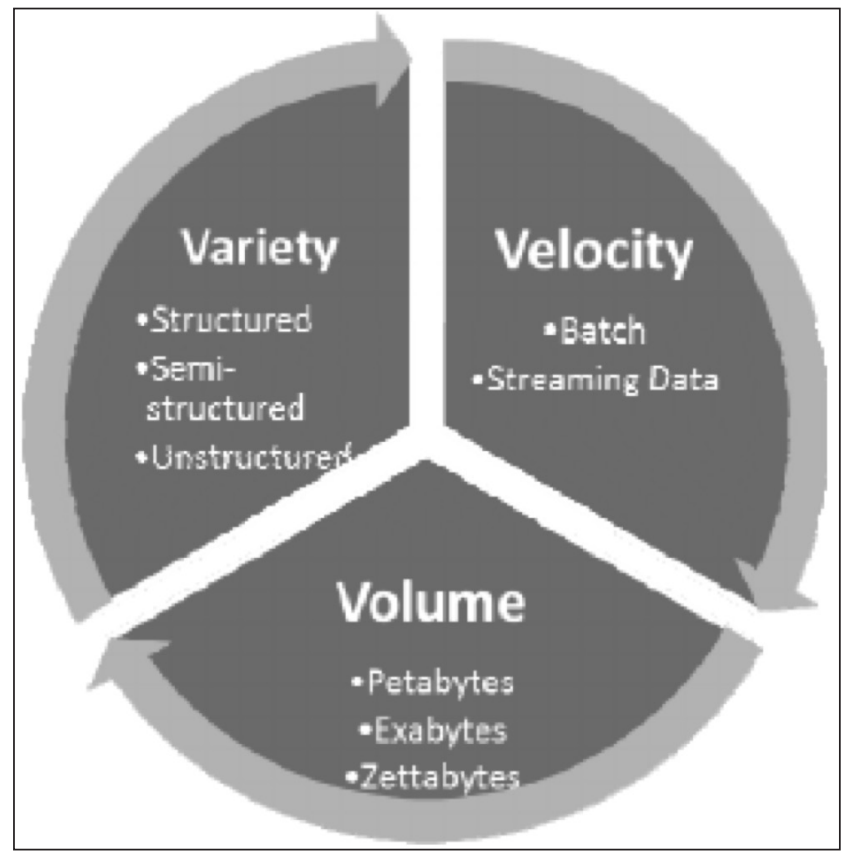

Figura 9: L'architettura del modello a 3V per Big Data.

Il cosiddetto «Garnter Hyper Cycle» raffigurato in figura 10 ben rappresenta le fasi di vita e di evoluzione delle scoperte scientifiche nel tempo e in particolare il fatto che per i «big data» oggi abbiamo raggiunto quasi il picco della cosiddetta "aspettativa tecnologica».

E' immediato intuire come uno degli aspetti piu' «delicati» del contributo dei BIG DATA alla conoscenza biomedica riguardi quella che molti definiscono la quarta «V» ovvero la «veracity», la veridicità: quanta «buona informazione» si puo' dedurre dai «Big data «? Quanto è possibile utilizzare questi dati raccolti senza alcun disegno sperimentale e sulla cui qualità ci sono poche informazioni, per trarre conclusioni in termini «causali» 
su patologie o fattori di rischio, aspetti già complessi da trattare in tutti gli studi osservazionali?

L'utilizzo dei BIG Data nelle scienze biomediche è diventato materia molto attuale anche dal punto di vista della sanità pubblica e della bioetica. Nel 2015 il Nuffield Council on Bioethics ha dedicato una intera sezione al tema "The collection, linking and use of data in biomedical research and health care: ethical issues». In questo contesto è emerso chiaramente come ci sia crescente interesse pubblico per un uso responsabile dei dati che generi conoscenza, promuova l'innovazione e migliori la salute. A questo si accompagnano le legittime preoccupazioni riguardo alla privacy. Emerge quindi una

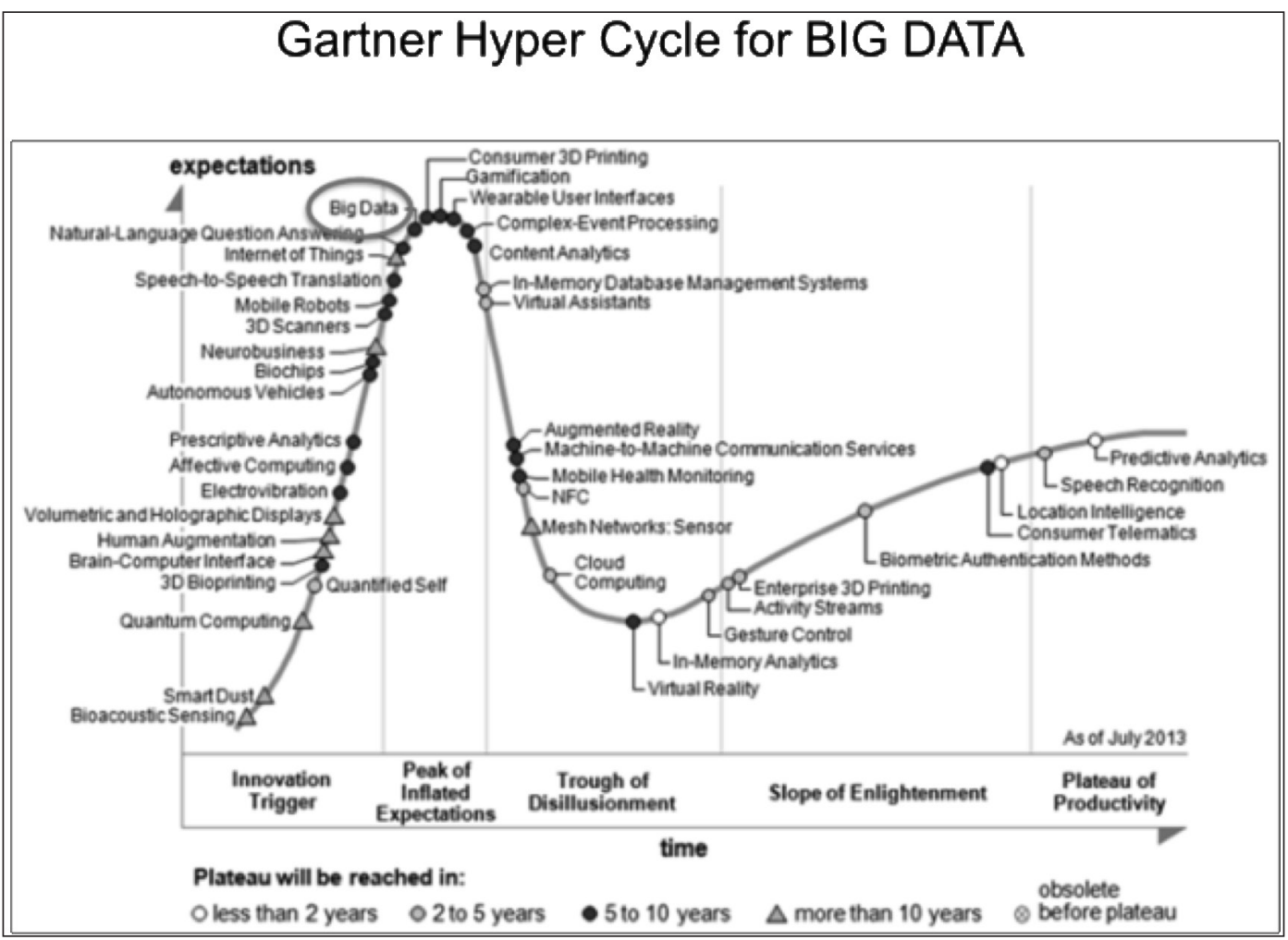

Figure 10: Gartner Hype Cycles provide a graphic representation of the maturity and adoption of technologies and applications, and how they are potentially relevant.

serie di criticità legate alla trasparenza, alla privacy, al consenso informato, $i$, che dovrebbero essere adeguatamente affrontate per fare in modo di gestire al meglio e valorizzare questa enorme disponibilità di dati e tutti i cambiamenti che ne deriveranno a livello di conoscenza globale. A partire dal 2012, ogni giorno vengono generati circa 2,5 exabyte (numero che dovrebbe raddoppiare nei prossimi anni) e che un uso improprio di questa enorme mole di dati potrebbe rivelare correlazioni con malattie tali da far ritenere una persona non adatta a determinati lavori piuttosto che ad ottenere determinate condizioni per le assicurazioni sanitarie o sulla vita. Nasce infatti quella che viene definita "digital epidemiology» che consiste in una nuova generazione di sistemi di sorveglianza di sanità pubblica che operano attraverso i confini internazionali e integrano i sistemi tradizionali, sfruttando l'accesso diffuso a Internet e la crescita esplosiva dei dispositivi mobili e delle piattaforme di condivisione online. Utilizzando i dati globali in tempo reale,

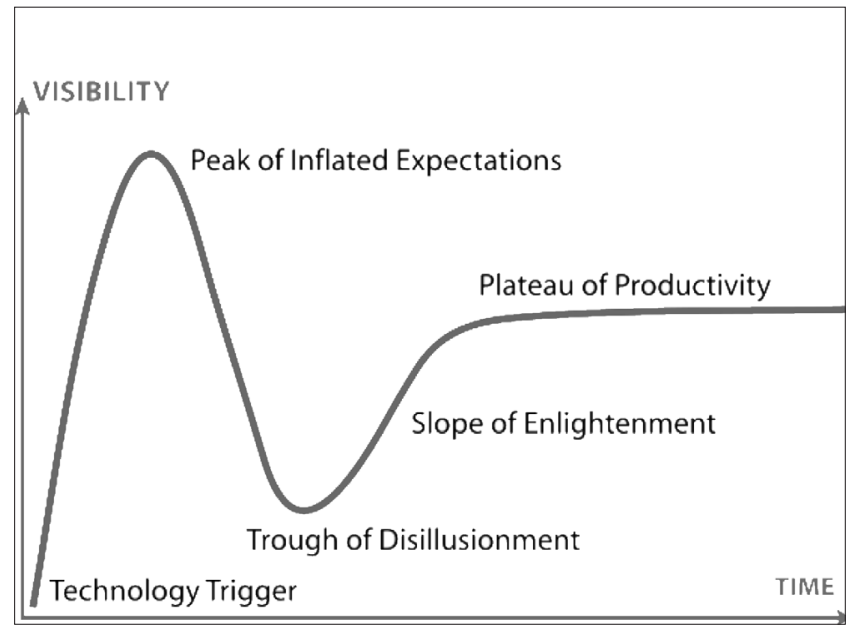

l'epidemiologia digitale promette ad esempio di rilevare tempestivamente il focolaio di una malattia. Il caso più recente è l'epidemia di Ebola in Africa occidentale del 2014 , ovvero di fare in tempi molto piu' rapidi quello che John Snow nel 1854 ha fatto per scoprire il colera impiegando mesi di lavoro.

\section{Conclusioni}

Le tecnologia del «dato « è progredita parallelamente alle tecnologie biomediche rendendo trasformando il concetto di analisi epidemiologica, analisi predittiva e surveillance parti integrate di un unico processo alla base della prevenzione e degli interventi di sanità pubblica.

Così come la medicina di precisione impiega dati sui singoli pazienti per pianificare l'intervento terapeutico più adatto, così la prevenzione di precisione fa uso dei «Big Data» per studiare l'impatto dei fattori di rischio 
sullo sviluppo di determinate patologie all'interno di una popolazione e per identificare strategie atte a prevenirne l'insorgenza o per ottimizzare l'uso delle pratiche e delle risorse terapeutiche esistenti.

E' necessari quindi focalizzarsi a livello di policy sulle strategie di prevenzione che i medici, gli epidemiologi, i genetisti, le istituzioni sanitarie, potrebbero mettere in atto avendo accesso, incrociando e facendo dialogare tra loro questo enorme volume di dati ad alta velocità, complessità e variabilità». Inoltre questa grossa mole di dati generata su singoli individui potrebbe creare problemi etici e giuridici, di cui bisogna tener conto al momento di pianificare studi di popolazione. Se quindi è vero che dalla integrazione delle diverse fonti di dati possano nascere apporti fondamentali per l'individuazione dei fattori di rischio, di nuovi biomarcatori, di focolai di infezioni legati a nuovi virus, tuttavia la valutazione del comportamento e degli atteggiamenti di salute delle persone e la farmacovigilanza restano di interesse primario.

\section{Bibliografia}

de Moivre, A. (1725). Annuities upon Lives: or, The Valuation of An nuities uon any Number of Lives; as also, of Reversions. To which is added, An Appendix concerning the Expectations of Life, and Probabilites of Survivorship. London: Fayram, Motte and Pearson.

Eurostat (2006). EU integration seen through statistics. Luxembourg: Publications of the European Union. http://epp.eurostat.ec.europa. eu/cache/ITY_OFFPUB/KS-71-05-691/EN/KS-71-05-691-EN.PDF (18.03.2013).

Gompertz, B. (1825). On the nature of the function expressive of the law of human mortality. Philosophical Transactions of the Royal Society of London, Series A 115, 513-580.

Hald, A. (1990). A History of Probability and Statistics and Their Applications before 1750. New York: Wiley.

Makeham, W.M. (1860). On the law of mortality, and the construction of mortality tables. Journal of the Institute of Actuaries, 8, 301

Makeham, W.M. (1860). On the law of mortality, and the construction of mortality.
Neff, G. (2013). Why big data won't cure us. Big Data, 1, 117-123. pmid:25161827.

Salathé, M., Bengtsson, L., Bodnar, T.J., Brewer, D.D., Brownstein, J.S. et al. (2012). Digital epidemiology. PLoS Comput Biol, 8: e1002616. pmid:22844241.

Stjernsward, J. (WHO) (1989). Battle against tobacco. Editorial. Journal of the National Cancer Institute, 81,1524-1525.

United Nations Economic Commission for Europe (2010). Regional Ministerial Conference on Ageing will review 10 years of implementation of Madrid International Plan of Action on Ageing. Genf: UNECE. http://www.unece.org/press/pr2010/10env_p40e.html (19.07.2013).

Vayena, E., Salathé, M., Madoff, L.C. \& Brownstein J.S. (2015). Ethical Challenges of Big Data in Public Health. PLoS Comput Biol, 11(2): e1003904.

\section{Autore}

Professore Clelia Di Serio, Ordinario di Statistica Medica ed Epidemiologia presso Unisr. 1991: Laurea in Economia con indirizzo statistico. 1995; Executive Master in Statistics (UNC-USA) 1992-1996. Dottorato in Statistica ( Chapel Hill, PhD program University of North Carolina-USA joint with University of Trento). Dal 2007 Professore incaricato di Statistica presso l'Università degli Studi della Svizzera Italiana sede Lugano. Dal 2005 Direttore del CUSSB (University Centre for Statistics in the Biomedical Sciences). Pubblicazioni: http:// www.unisr.it/k-teacher/diserio-mariacleliastefania/\#14568419 75415-dfc853c9-c0d3.

\section{Contatto}

diserio.clelia@hsr.it

clelia.di.serio@usi.ch

Centro Universitario di Statistica per le Scienze Biomediche (CUSSB: www.cussb.unisr.it)

Università Vita-Salute San Raffaele

Via Olgettina 58

20132 Milano

Università della Svizzera Italiana, Via Buffi, Lugano. 